\title{
Simultaneous Selective Thoracic Fusion of Lenke-1C Scoliosis and Reduction of Symptomatic Spondylolisthesis: A Case Report
}

\author{
Ruffilli Alberto, Fiore Michele, Barile Francesca, Pasini Stefano, Viroli Giovanni, Manzetti Marco, Faldini Cesare
}

IRCCS Istituto Ortopedico Rizzoli (IOR), Bologna, Italy

Corresponding Author:

Fiore Michele, MD

ORCID: 0000-0003-0546-670X

via G.C. Pupilli 1, Bologna, 40136, Italy

Tell: +393405219768 ,

Fax: +390516366669

E-mail: michele.fiore@ior.it

Received: November 21, 2020

Revised: January 24, 2021

Accepted: February 07, 2021
The combined treatment of unrelated Lenke-1C curves and spondylolisthesis represents a challenge: The two arthrodesis areas must achieve corrections while preserving mobility as much as possible. We reported a case of 20-year-old girl with Lenke-1C scoliosis and Meyerding grade-2 symptomatic L5-S1 isthmic spondylolisthesis. She was treated with one-stage correction with T3-T12 posterior selective thoracic fusion (STF) associated to reduction and fusion of the spondylolisthesis. Pre-op Cobb angle of the main thoracic (MT) curve was $62^{\circ}$. The non-structural lumbar $(\mathrm{L})$ curve was $52^{\circ}$. Coronal imbalance was $39 \mathrm{~mm}$. 1-month post-op $\mathrm{X}$-ray showed a reduction of MT-curve to $32^{\circ}$ and L-curve to $24^{\circ}$. The coronal imbalance was $13 \mathrm{~mm}$. A satisfactory sagittal alignment and olisthesis reduction were achieved. At 24-months follow-up, L-curve increased to $30^{\circ}$. Coronal imbalance was $24 \mathrm{~mm}$. Loss of correction appeared stable at 36-months final follow-up. Although the evidence cautiously suggests STF to treat also Lenke-1C scoliosis, this case confirmed that the risk of worsening coronal decompensation exists, and it is possibly increased by a distal lumbar arthrodesis to treat spondylolisthesis. However, we believe that STF is justified as worsening L-curve does not balance the possibility of preserving motility of the lumbar tract, also because the need for revision is an uncommon event.

Key Words: Lenke-1C scoliosis, Spondylolisthesis, Spine deformity, Selective thoracic vertebral arthrodesis

\section{INTRODUCTION}

The concomitant occurrence of scoliosis and spondylolisthesis is retrieving in $15-48 \%$ of cases $^{14)}$. This association is etiologically classifiable in three groups: type-1: unrelated thoracic idiopathic scoliosis and lumbar spondylolisthesis; type-2: antalgic low-grade spasm scoliosis secondary to spondylolisthesis; type-3: lumbar scoliosis secondary to an asymmetric olisthetic defect ${ }^{2)}$.

Type-1 is reported in $6.2 \%$ of patients with idiopathic scoliosis ${ }^{5}$. The combined treatment represents a challenge with Lenke-1C curves: the two arthrodesis areas must achieve corrections while preserving mobility as much as possible.

\section{CASE REPORT}

A 20-year-old girl presented low-back pain with bilateral L5 radicular pain, unresponsive to conservative therapies. X-ray showed a Meyerding grade-2 symptomatic high-dysplastic L5-S1 isthmic spondylolisthesis (Figure 1), with concomitant Lenke-1C scoliosis. Cobb angle of the main thoracic (MT) curve was $62^{\circ}$. The non-structural lumbar $(\mathrm{L})$ curve was $52^{\circ}$, resulting highly flexible $\left(19^{\circ}\right.$ at side-bending $\mathrm{X}$-ray). Standing $\mathrm{X}$-ray showed coronal imbalance: C7 plumb-line (C7PL)/central sacral vertical line (CSVL) distance was $39 \mathrm{~mm}$ (Figure 2). Previous follow-up X-rays had confirmed that both scoliosis and spondylolisthesis were progressive during the conservative treatment with TLSO Brace (Thoracic Lumbar Sacral Orthosis).

We performed one-stage correction of both deformities: T3-T12 selective thoracic spine fusion (STF) associated to reduction and fusion of the slipped vertebra.

The first incision was from T3 to T12. To increase spinal flexibility three apical Ponte osteotomies were performed. High-density uniplanar pedicle screws were placed with straight-forward Funnel technique. A translation maneuver was applied adopting two asymmetric $5.5 \mathrm{~mm}$ cobalt-chrome rods. Rods were overshaped on the desired kyphosis apex (T6) to obtain a lifting effect 
restoring kyphosis. At the scoliosis apex, the rod on the concave side was over-shaped and the rod on the convex side was under-shaped, to obtain a rotational effect achieving deformity correction. Direct vertebral rotation was applied to complete correction ${ }^{4)}$.

Through a second incision, two polyaxial reduction screws were placed in the L5 pedicles and two polyaxial screws in S1. The posterior mobile arch was removed in block; discectomy was performed, and the slippage was reduced. Two titanium cages of appropriate size were placed.

1-month post-operative $\mathrm{X}$-ray showed a reduction of MT-curve to $32^{\circ}$ and L-curve to $24^{\circ}$. The coronal imbalance was reduced to $13 \mathrm{~mm}$. A satisfactory sagittal alignment and olisthesis reduction were achieved (Figure 3). At 24-months follow-up, X-ray showed an increase of L-curve to $30^{\circ}$. C7PL/CSVL distance was $24 \mathrm{~mm}$ (Figure 4). No further loss of correction appeared at 36months follow-up.

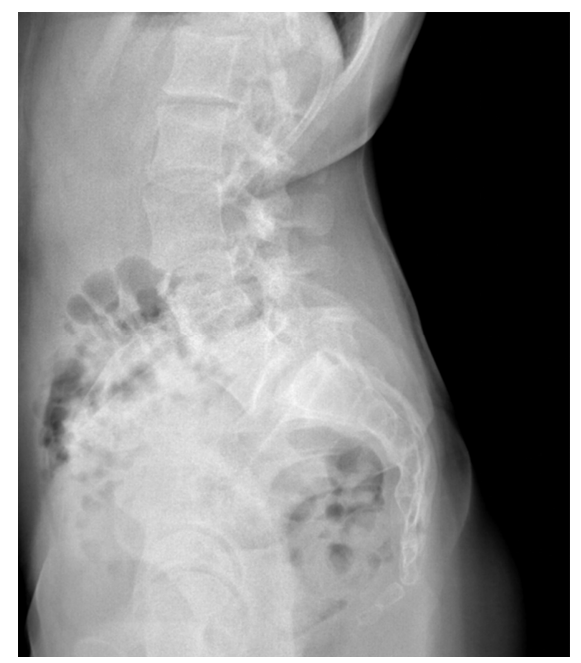

Figure 1. High dysplasia L5-S1 spondylolisthesis.

\section{DISCUSSION}

When Lenke classification was developed ${ }^{11)}$, the intent of the Authors not only was to describe AIS curve types, but also to help the surgeons deciding specific vertebral levels to be included in spinal arthrodesis. According to the Authors, the major curve has the largest Cobb angle and should always be included in the fusion. Whether or not the minor curves should be fused depends on their flexibility and how the deformity affects the sagittal plane ${ }^{11)}$ : if a minor curve corrects to $<25^{\circ}$ on coronal side-bending films and if, in addition, the kyphosis between $\mathrm{T} 2-\mathrm{T} 5$ and $\mathrm{T} 10-\mathrm{L} 2$ is $<20^{\circ}$, the curve is regarded as being non-structural and does not have to be included in the fusion because spontaneous coronal correction after selective fusion of the major curve is expected ${ }^{9}$. . Therefore, STF can be performed when both the thoracic and the TL/L curves deviate from the midline, but only the major curve is fused, leaving the minor curve(s) unfused and mobile ${ }^{3)}$. This procedure has been proven successful in Lenke-1 and Lenke-2 curves $^{6,9)}$ and in Lenke-3 curves when specific criteria are met (MT:TL/L Cobb, AVT-MT: AVT$\mathrm{TL} / \mathrm{L}$ and AVR-MT:AVR-TL/L >1.2 ${ }^{3)}$. However, Lenke-1C curves treated with STF have a higher risk of post-operative coronal decompensation than $A / B^{3,8,10)}$. Kwan et al., about STF outcome, stated the risks of coronal decompensation of $20.5 \%$, lumbar decompensation of $9.1 \%$, adding-on phenomenon of $25.0 \%{ }^{10}$ ).

Coronal imbalance can lead to aesthetic concerns, rarely to the need to extend the fusion ${ }^{10,12,15)}$. While the main causes of immediate post-operative decompensation (IPCD) following STF have been widely investigated (pre-operative coronal decompensation, excessive MT-curve correction, inappropriate selection of LIV), less is known about spontaneous correction of L-curves $^{7,13,16)}$. It may be attributed to postural reflexes, potentially existing in the relatively flexible non-structural curves. Ishikawa et al. reported STF with LIV proximal to SV leads less frequently to IPCD, but it results in lower correction of the

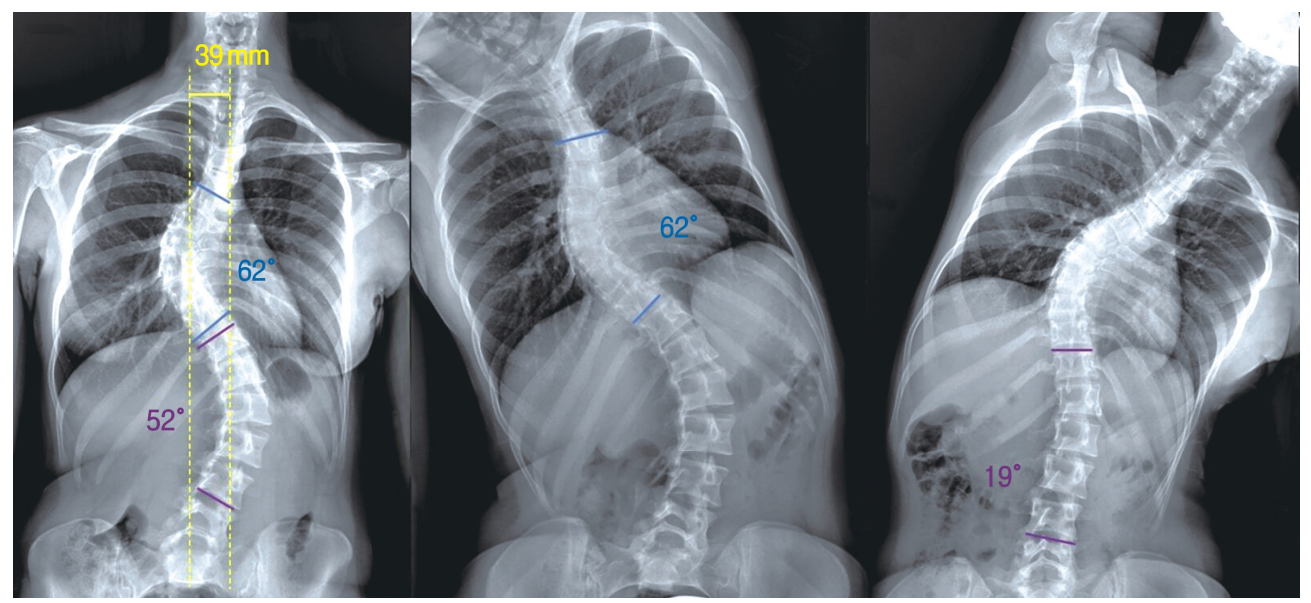

Figure 2. AP pre-operative $X$-ray, right and left bending. Coronal C7PL-CSVL distance (yellow), MT-curve Cobb (blue), L-curve Cobb (purple). The $\mathrm{X}$-rays show a Lenke-1C curve, with a highly structured MT-curve and a flexible L-curve. C7PL/CSVL distance was $39 \mathrm{~mm}$, resulting in a severe coronal imbalance. 


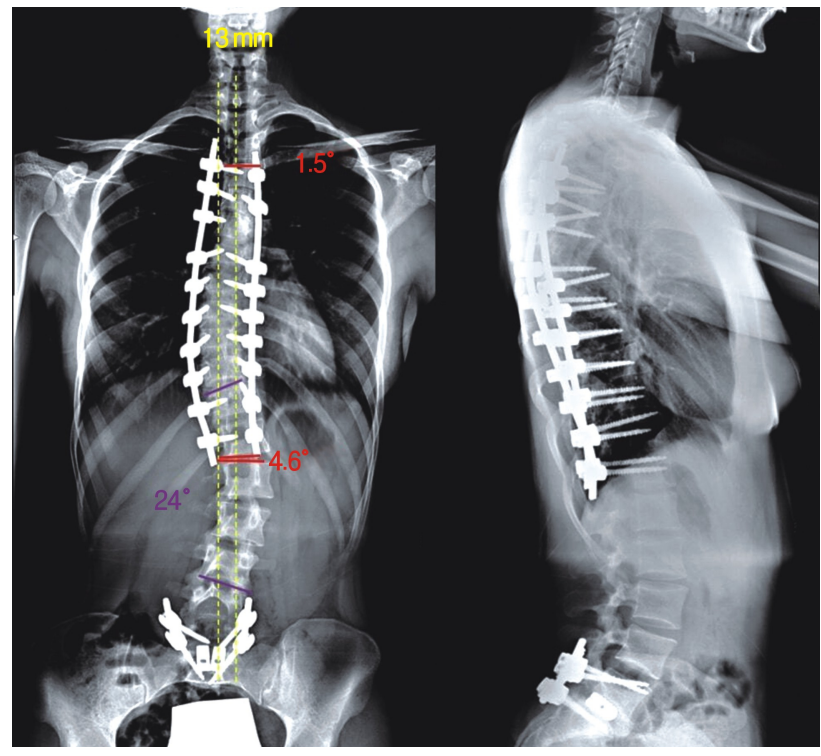

Figure 3. 1-month follow-up AP and sagittal pre-operative $X$-rays. Coronal C7PL-CSVL distance (yellow), MT-curve Cobb (blue), L-curve Cobb (purple), UIV- and LIV-tilt (red). After combined arthrodesis (correction of scoliosis with T3-T12 STF + reduction and fusion of L5-S1 spondylolisthesis), both MT- and L-curve are significantly reduced, with also an improvement in coronal alignment. On sagittal plane an excellent alignment was obtained with stable L5-S1 reduction.

non-instrumented L-curve ${ }^{7)}$. Conversely, not-selective fusion with LIV distal to SV would result more frequently in IPCD, but with a higher trend to progressive L-curve recompensation. Nevertheless, patients with coronal decompensation at final follow-up all had IPCD, and none of the post-operatively compensated patients resulted decompensated at final follow-up.

In the presented case, considering the curve type, the age of the patient, the L5-S1 arthrodesis, and wanting to preserve as much movement as possible, the Authors chose to treat the Lenke-1C scoliosis with STF. In fact, despite the higher risk of coronal decompensation when compared to Lenke-1A and $B$ curves, STF has been demonstrated as an effective treatment for the so-called "false double major" curve (Lenke-1C/King II)". Moreover, STF maintains the option to extend the fusion to the lumbar spine when the non-instrumented curve is found to be progressing. Hypothetically, the choice of LIV was intended to balance the risk of IPCD without sacrificing the progressive coronal compensation capacity of the L-curve. However, postoperative X-ray showed a certain degree of IPCD (despite an improvement from pre-operative condition), but coronal misalignment progressively increased at the 3-, 6- and 12-months follow-up, remaining stable up to the final 3-years follow-up.

The indication to perform a combined one-staged surgery in the patient was driven by the evidence that both pathological conditions were already progressing and worsening, as well as clearly symptomatic. We considered that both interventions had to be performed, so we avoided a two-staged surgery (first the scoliosis correction and then the spondylolisthesis, or vice versa) in order not to risk further worsening of the pathology

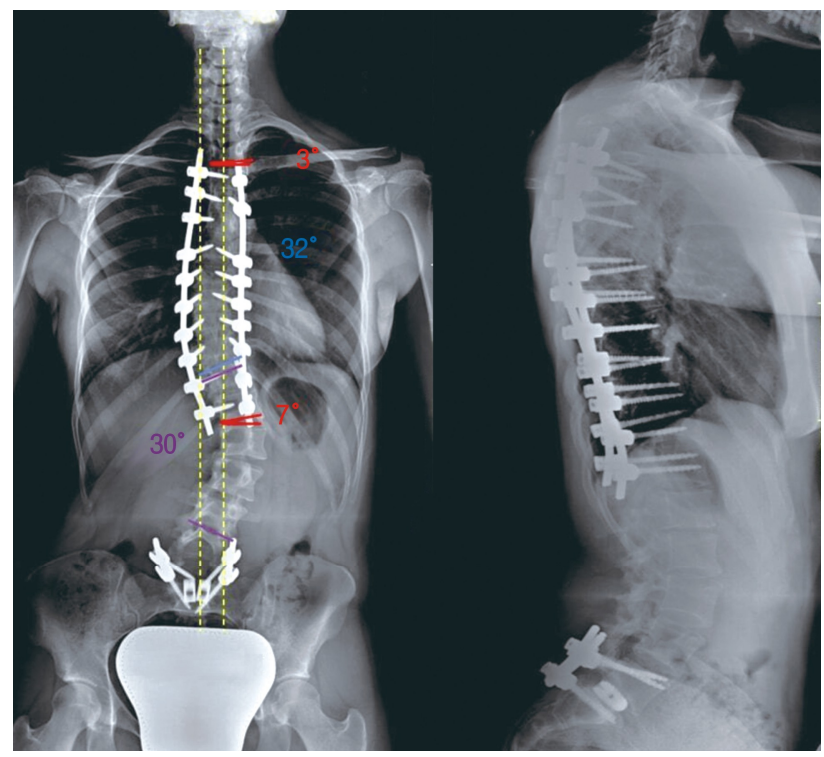

Figure 4. 3-years follow-up AP and sagittal pre-operative $X$-rays. Coronal C7PL-CSVL distance (yellow), MT-curve Cobb (blue), L-curve Cobb (purple), UIV- and LIV-tilt (red). A mild worsening of the coronal imbalance and a L-curve adding-on phenomenon were seen, although the loss of correction appeared stable compared with the 2-year follow-up. On sagittal plane no loss of correction was found at final follow-up.

not surgically treated, caused by the biomechanical changes secondary to the first intervention. Obviously also the possibility to avoid a second general anaesthesia and a second rehabilitation program contributed to our decision.

This case supports our opinion that distal lumbar arthrodesis, by tightening the L-curve reducing its compensation potential, may represent a risk-cofactor for the occurrence of lumbar adding-on following STF. This should be considered in planning the arthrodesis area in similar cases. However, we believe that STF is justified as worsening the lumbar curve does not balance the possibility of presenving the motility of the lumbar tract, also because the need for revision is an uncommon event for these issues.

\section{CONCLUSIONS}

In conclusion, although the evidence cautiously suggests STF to treat also Lenke-1C scoliosis, the risk of worsening coronal decompensation and adding-on exists and it is possibly increased by association with spondylolisthesis. In Authors' opinion, this report adds useful elements in the choice of treatment in a challenging scenario such as the case presented.

\section{CONFLICT OF INTEREST}

No potential conflict of interest relevant to this article.

\section{REFERENCES}

1. Crawford $\mathrm{CH} 3^{\text {rd }}$, Lenke LG, Sucato DJ, Richards BS $3^{\text {rd }}$, Emans 
JB, Vitale MG, Erickson MA, Sanders JO: Selective thoracic fusion in Lenke-1C curves: Prevalence and criteria. Spine (Phila Pa 1976). Jul;15;38(16):1380-1385, 2013

2. Crostelli M, Mazza O: AIS and spondylolisthesis. Eur Spine J Mar;22 Suppl 2(Suppl 2):S172-184, 2013

3. Edwards CC $2^{\text {nd }}$, Lenke LG, Peelle M, Sides B, Rinella A, Bridwell $\mathrm{KH}$ : Selective thoracic fusion for adolescent idiopathic scoliosis with C modifier lumbar curves: 2- to 16-year radiographic and clinical results. Spine (Phila Pa 1976). Mar;1;29(5):536-546, 2004

4. Faldini C, Perna F, Geraci G, Pardo F, Mazzotti A, Pilla F, et al.: Correction to: Triplanar correction of adolescent idiopathic scoliosis by asymmetrically shaped and simultaneously applied rods associated with direct vertebral rotation: Clinical and radiological analysis of 36 patients. Eur Spine J Jan;28(1):196, 2019

5. Fisk JR, Moe JH, Winter RB: Scoliosis, spondylolysis, and spondylolisthesis. Their relationship as reviewed in 539 patients. Spine (Phila Pa 1976). Sep;3(3):234-245, 1978

6. Frez R, Cheng JC, Wong EM: Longitudinal changes in trunkal balance after selective fusion of King II curves in adolescent idiopathic scoliosis. Spine (Phila Pa 1976) Jun;1;25(11):1352-1359, 2000

7. Ishikawa M, Cao K, Pang L, Fujita N, Yagi M, Hosogane N, et al.: Onset and remodeling of coronal imbalance after selective posterior thoracic fusion for Lenke-1C and 2C adolescent idiopathic scoliosis (a pilot study). Scoliosis Spinal Disord May 12: 12:16, 2017

8. Ishikawa M, Nishiyama M, Kamata M: Selective Thoracic Fusion for King-Moe Type II/Lenke-1C Curve in Adolescent Idiopathic Scoliosis: A Comprehensive Review of Major Concerns. Spine Surg Relat Res Oct;10;3(2):113-125, 2018

9. King HA, Moe JH, Bradford DS, Winter RB: The selection of fusion levels in thoracic idiopathic scoliosis. J Bone Joint Surg
Am Dec;65(9):1302-1313, 1983

10. Kwan MK, Chiu CK, Tan PH, Chian XH, Ler XY, Ng YH, et al.: Radiological and clinical outcome of selective thoracic fusion for patients with Lenke-1C and 2C adolescent idiopathic scoliosis with a minimum follow-up of 2 years. Spine J Dec;18(12): 2239-2246, 2018

11. Lenke LG, Betz RR, Harms J, Bridwell KH, Clements DH, Lowe TG, Blanke K: Adolescent idiopathic scoliosis: A new classification to determine extent of spinal arthrodesis. J Bone Joint Surg Am Aug;83(8):1169-1181, 2001

12. Schulz J, Asghar J, Bastrom T, Shufflebarger H, Newton PO, Sturm P, et al.: Optimal radiographical criteria after selective thoracic fusion for patients with adolescent idiopathic scoliosis with a C lumbar modifier: Does adherence to current guidelines predict success? Spine (Phila Pa 1976) Nov;1;39(23):E1368E1373, 2014

13. Seitsalo S, Osterman K, Hyvãrinen H, Tallroth K, Schlenzka D, Poussa M: Progression of spondylolisthesis in children and adolescents. A long-term follow-up of 272 patients. Spine (Phila Pa 1976) Apr;16(4):417-421, 1991

14. Seitsalo S, Osterman K, Poussa M: Scoliosis associated with lumbar spondylolisthesis. A clinical survey of 190 young patients. Spine (Phila Pa 1976). Aug;13(8):899-904, 1988

15. Studer D, Awais A, Williams N, Antoniou G, Eardley-Harris N, Cundy P: Selective fusion in adolescent idiopathic scoliosis: A radiographic evaluation of risk factors for imbalance. J Child Orthop Apr;9(2):153-160, 2015

16. Wang WJ, Yeung HY, Chu WC, Tang NL, Lee KM, Qiu Y, Burwell RG, Cheng JC: Top theories for the etiopathogenesis of adolescent idiopathic scoliosis. J Pediatr Orthop Jan-Feb;31(1 Suppl): S14-27, 2011 